\title{
Seed Magnetic Fields Generated by Primordial Supernova Explosions
}

\author{
Oswaldo D. Miranda, Merav Opher and Reuven Opher \\ Instituto Astronômico e Geofísico - Universidade de São Paulo \\ Av. Miguel Stéfano 4200, São Paulo, 04301-904, SP, Brazil
}

\begin{abstract}
The origin of the magnetic field in galaxies is an open question in astrophysics. Several mechanisms have been proposed related, in general, with the generation of small seed fields amplified by a dynamo mechanism. In general, these mechanisms have difficulty in satisfying both the requirements of a sufficiently high strength for the magnetic field and the necessary large coherent scales. We show that the formation of dense and turbulent shells of matter, in the multiple explosion scenario of Miranda and Opher $(1996,1997)$ for the formation of the large-scale structures of the Universe, can naturally act as a seed for the generation of a magnetic field. During the collapse and explosion of Population III objects, a temperature gradient not parallel to a density gradient can naturally be established, producing a seed magnetic field through the Biermann battery mechanism. We show that seed magnetic fields $\sim 10^{-12}-10^{-14} G$ can be produced in this multiple explosion scenario on scales of the order of clusters of galaxies (with coherence length $L \sim 1.8 \mathrm{Mpc}$ ) and up to $\sim 4.5 \times 10^{-10} \mathrm{G}$ on scales of galaxies $(L \sim 100 \mathrm{kpc})$.
\end{abstract}

Key words: magnetic fields - Cosmology: theory - early Universe - large-scale structure of Universe.

\section{INTRODUCTION}

The origin of primordial magnetic fields is one of the most important problems in astrophysics (Peebles 1993) and, as pointed out by Barrow et al. (1997), is still a mystery. Various processes have been proposed for the primordial origin of a seed field. In particular, several mechanisms on small dimensions have been suggested such as fluctuations in the primordial plasma, creation in phase transitions in the inflation era, etc. However, no satisfactory theory has been proposed which creates a sufficiently intense magnetic field on the large scales of galaxies and clusters of galaxies.

The basic idea of a seed field is that, once produced, it can be later amplified by a dynamo process (e.g. Zel'Dovich et al. 1983). However, it is necessary to generate a seed field with a sufficient intensity such that a dynamo process can amplify it to the present observed values. Another aspect that should be satisfied by the proposed mechanism is that the magnetic field should have a sufficiently large coherence length so that it can be amplified by the dynamo mechanism (e.g., be at least of galactic dimension such that the differential galactic rotation can amplify it).

Harrison (1973) proposed a mechanism for the generation of magnetic fields before the recombination epoch. Assuming the existence of turbulence with nonzero vorticity, he arrived at a magnetic field $\sim 10^{-18} G$ on galactic scales. Rees (1987) pointed out, however, that the significant vorticity required by the model of Harrison is diffi- cult to reconcile with the expected predominance of irrotational density perturbations that should become dominant in the post-recombination epoch. Another mechanism suggested in the plasma epoch, but without requiring vorticity, was proposed by Tajima et al. (1992). Based on the fluctuation-dissipation theorem, they studied the electromagnetic fluctuations present in plasma in thermal equilibrium and showed that in the plasma epoch $\left(1 s<\tau<10^{13} s\right.$, where $\tau$ is the cosmic time), a high intensity magnetic field exists at $\omega \sim 0$ (i.e., zero frequency). It is difficult to exactly quantify the present intensity of this magnetic field, but it could be as high as $10^{-12} \mathrm{G}$. However, a more detailed treatment of the plasma processes by Opher and Opher (1997a, 1997b) showed that the magnetic field at zero frequency is appreciably less than that predicted by Tajima et al. (1992).

Some authors suggested that magnetic fields could be created during inflation (Turner and Widrow 1988; Ratra 1992). These scenarios can lead to sufficiently large coherence scales for the fields because inflation can lead to mechanisms acting on horizon scales. However, the predicted amplitudes are extremely sensitive to the parameters used. Another suggestion is the creation of magnetic fields in phase transitions. Quasnock et al. (1988) proposed a seed field generating mechanism during the quark-hadron phase transition (assuming a first order transition). The field strength obtained is $\sim 10^{-17} \mathrm{G}$ on a scale $\sim 10^{10} \mathrm{~cm}$ at the recombination epoch. This scale is of order $1 A U$ at the present time, very small compared to galactic scales. More recently, 
Sigl et al. (1997) obtained for the seed field, generated in a first order phase transition, strengths $\sim 10^{-29} G$ on a $10 \mathrm{Mpc}$ scale for the electroweak transition and $10^{-20} \mathrm{G}$ for the QCD transition.

The generation of primordial magnetic fields in the early Universe, as a consequence of the electroweak phase transition, has been studied more recently by Törnkvist (1997, 1998) and Olesen (1997). In particular, Olesen showed that at the electroweak scale, considering that the magnetic field is essentially random, it is necessary to produce a field $<B>\sim 10^{15} G$ such that at the present time we have a primordial magnetic field of order $10^{-15} \mathrm{G}$ on a scale of $100 \mathrm{kpc}$.

Barrow et al. (1997) has obtained, through a statistical analysis of the 4-year COBE data, an upper limit of $B_{0}<3.4 \times 10^{-9}\left(\Omega_{0} h_{50}^{2}\right)^{1 / 2} G$ for the present strength of any primordial homogeneous magnetic field. This limit is important because it restricts the value of the seed field during the pre-recombination epoch.

Opher and Wichoski (1997) suggested that the origin of the magnetic field is due to a nonminimal electromagneticgravitational coupling. Assuming that the magnetic dipole moment is proportional to the angular momentum with the proportionality constant normalized to existing observations, Opher and Wichoski (1997) obtained galactic magnetic fields comparable to those observed $(\stackrel{\sim}{\sim} \mu G)$.

For stars, a totally spontaneous mechanism of generating magnetic fields, not requiring seed fields, was suggested. It is the Biermann mechanism (Biermann 1950) that depends on the differential rotation in stars. As pointed out by Kemp (1982), what creates the Biermann effect is the difference in the acceleration imparted to the electrons, as compared to the ions, by the differential pressures. The difference in acceleration results in currents generating a double toroidal magnetic field, with the magnetic fields being oppositely circulating in the upper and lower hemisphere (the upper and lower hemispheres are defined with respect to the rotation axis).

Several works deal with this mechanism (e.g., Mestel and Roxburgh 1962 and Roxburgh 1966). In particular, Roxburgh (1966) analyzed whether or not there is a meridional circulation in stars.

Recently, Kulsrud et al. (1997) suggested a mechanism of generating protogalactic magnetic fields by a Biermann type like mechanism on shocks in the intergalactic medium. Their mechanism generates a seed field of $10^{-21} \mathrm{G}$.

We present here an alternative model to generate a seed magnetic field of magnitude $\sim 10^{-14} G$ to $5 \times 10^{-10} G$ which is many orders of magnitude greater than many of the previously suggested scenarios. It also is created over a relatively large scale making it relatively easy to amplify by galactic differential rotation. It is based on the supernovae explosion model of Miranda and Opher (1996, 1997) for large-scale structure formation. Whereas, for example, the Kulsrud et al. (1997) scenario requires a very strong dynamo action to amplify the seed magnetic field of $\sim 10^{-21} G$ to presently observed values of $\sim 10^{-6} G$, our scenario requires only a relatively mild amplification.

The mechanism here proposed for generating seed magnetic fields is based on the study of Lazarian (1992). It is a Biermann type mechanism because it requires that the electronic temperature and density gradients are not parallel, similar to what happens in the original mechanism in ro- tating stars. We coupled this Biermann type mechanism to the explosion model of Miranda and Opher $(1996,1997)$ for structure formation.

The multiple explosion scenario here presented is an extension of the model first studied by Ostriker and Cowie (1981) and Ikeuchi (1981). Several extensions of the Ostriker-Cowie-Ikeuchi model were posteriorly made (e.g., Carr, Bond and Arnett 1984, Carr and Ikeuchi 1985). In particular, Carr and Ikeuchi (1985) studied a hierarchical model composed of the evolution of discreet explosive stages. After the first results of the COBE satellite, this model was considered incapable to produce large structures without violating the limit of the anisotropy observed in the cosmic background radiation. The multiple explosion scenario was recently retaken by Miranda and Opher $(1996,1997)$. They showed that, within the limits of their model, the multiple explosion scenario can produce large voids with Compton-y distortions compatible with the COBE limits.

In $\S 2$, we show that it is possible to generate seed magnetic fields $\sim 10^{-12}-10^{-14} G$ on scales of galactic clusters and in $\S 3$ up to $\sim 4.5 \times 10^{-10} G$ on $\sim 100 k p c$ scales. These seeds can be amplified during galactic formation to the observed values $\sim 1 \mu G$ by a dynamo mechanism. Our discussion and conclusions are presented in $\S 3$.

\section{THE MODEL}

When magnetic fields are present, we use magnetohydrodynamics to follow their effects. In this work we consider that the electrical conductivity is infinite. Thus, using Ohm's law in the Faraday equation, we have

$\frac{\partial \mathbf{B}}{\partial t}=\nabla \times(\mathbf{v} \times \mathbf{B})$

On the other hand, Miranda and Opher (1996, 1997) showed that the multicycle explosion scenario produces voids with characteristic radii $\sim 18-40 M p c$ (with the present value of the Hubble constant $h=0.5$, in units of $100 \mathrm{kms}^{-1} \mathrm{Mpc}^{-1}$ ). In this turbulent ambient medium formed by the explosions of Population III objects, we have a high probability for the formation of a temperature gradient not parallel to the density gradient.

The process proposed by Biermann (1950) is an efficient battery mechanism (compared with, for example, the battery mechanism based on Compton drag) because it can act on scales smaller than the galactic scale. Thus, we include an additional term in the anterior equation given by (see Lazarian 1992)

$\frac{\partial \mathbf{B}}{\partial t}=\nabla \times(\mathbf{v} \times \mathbf{B})+\frac{4 k_{B} c T}{\pi e}\left[\frac{\nabla n_{e}(r)}{n_{e}} \times \frac{\nabla T(r)}{T}\right]$

where $n_{e}$ is the electron density, $T$ is the temperature and $\mathbf{v}$ is the velocity of the shock front.

The second term on the right side of equation (2) is similar to the original Biermann mechanism, with the difference that we do not require rotation of the medium (as proposed by Biermann). It is worth stressing that an electromotive force can also be generated if there is a non-symmetric distribution of ions with different masses (e.g. the study made by Mestel and Roxburgh 1962). 
For a fluid in which shocks and photoheating can occur, temperature and density gradients are naturally not parallel so that $\nabla T \times \nabla n_{e} \neq \overrightarrow{\overrightarrow{0}}$. As shown by Miranda and Opher (1996, 1997), the multicycle explosive scenario for void formation produces a sequence of supernova explosions at high and moderate redshifts, beginning from the collapse of a Population III object.

At redshifts $z>8$, the main mechanism for cooling the shell of matter is the Compton cooling mechanism. This mechanism quickly produces a dense shell of matter where globules (that is, new gravitationally bound objects) are formed. These globules could be composed of stellar clusters of high mass, because the short Compton cooling time and the absence of metals favors the fragmentation of the globules into stars of high mass (that have a short life time). Thus, the explosion of these "stellar clusters" produces a turbulent medium where gradients of density and temperature which are not parallel can appear.

When the mass contained in a sphere of radius half the shell thickness is equal to the Jeans mass, objects (or globules) of mass $\delta m$ within the shell begin to collapse. The mass of these objects is obtained from

$\delta m=\left(\frac{M_{S}}{4 \pi R_{S}^{2} \xi}\right) \frac{4 \pi}{3}\left(\frac{\xi}{3}\right)^{3}$

where $\xi$ is the shell thickness, $M_{S}$ is the total mass contained inside the shell and $R_{S}$ is the radius of the shock front.

The temperature gradient exists inside the shell in the time interval from the explosion of the globules until the thermalization of the shell. Assuming that when the globules explode the characteristic separation distance between two globules is of the order of the shell thickness, we have

$\frac{\nabla n_{e}(r)}{n_{e}} \simeq \frac{\triangle n_{e}}{n_{e}} \frac{1}{\xi f_{n}}$

and

$\frac{\nabla T(r)}{T} \simeq \frac{\triangle T}{T} \frac{1}{\xi f_{T}}$

In Eqs. 4 and 5, the density gradient occurs over a distance $\xi f_{n}$ and the temperature gradient over a distance $\xi f_{T}$. In general, $f_{n}$ and $f_{T}$ are less than $1 / 2$ (and can be very much less). We then have $f_{n} f_{T} \equiv 0.25 F$ with $F \leqslant 1$. In the present investigation we consider that $F$ is in the interval 0.01 to 1 .

Thus, we obtain from equation (2)

$$
\frac{\partial \mathbf{B}}{\partial t}=\nabla \times(\mathbf{v} \times \mathbf{B})+\frac{16 k_{B} c T}{\pi e \xi^{2} F}\left[\frac{\triangle n_{e}}{n_{e}} \frac{\triangle T}{T} \sin (\phi)\right],
$$

where $\phi$ is the angle between the temperature and density gradients. The value $\phi=\pi / 2$ determines the upper limit to the seed magnetic field produced in the multiple explosion scenario. After the explosion of the globules, we assume that the strong shock condition is maintained. Thus, from the usual Rankine-Hugoniot relation, we have $\Delta n_{e} / n_{e}=4$. The temperature gradient occurs over the distance $\xi f_{T}$ in Eq. 5 and, over this distance, we may expect $\triangle T \sim T$ due to the fact that immediately after the explosion of the globules the temperature of the shock front is $\sim 10^{8} \mathrm{~K}$ while the temperature of the material external to the shock front is several orders of magnitude lower. Thus, we obtain in Eq. 6

$$
\frac{\partial \mathbf{B}}{\partial t}=\nabla \times(\mathbf{v} \times \mathbf{B})+\frac{64 k_{B} c T}{\pi e \xi^{2} F} .
$$

It is worth stressing that the angle $\pi / 2$ produces an upper limit to the seed magnetic field produced in this scenario, as mentioned above. On the other hand, when the globules are formed within the shell of matter, these objects have sufficiently small cross sections such that the external medium does not impede their propagation. The objects formed maintain their peculiar velocity $V_{P}=V_{S}-V_{H}$, where $V_{H}$ is the Hubble flow (see Miranda and Opher 1996). In this situation, when the explosion of the globules occurs, the density gradient is in the direction of the shell expansion, while the temperature gradient is situated between the globules (until the thermalization of the shell). In this case, the angle between the two gradients is not much smaller than $\pi / 2$.

When the globules are formed and begin to collapse, the first term on the right side of equation (7) is not active. On the other hand, during this phase of evolution, the magnetic field is frozen in the matter of the globules and (from the conservation of the magnetic flux) we need to include an additional term on the right side of equation (7) to take into account the variation with time of the area of the globules during the shell collapse. Thus

$\frac{\partial \mathbf{B}}{\partial t}=\nabla \times(\mathbf{v} \times \mathbf{B})+\frac{64 k_{B} c T}{\pi e \xi^{2} F}-\frac{2 \mathbf{B}}{\xi} \frac{d \xi}{d t}$.

It is worthwhile noting that the three terms on the right side of equation (8) act during well defined phases. The first term acts during the shell expansion phase. The second term acts during a short time after the explosion of the globules when a temperature gradient exists in the shell. The third term acts during the collapse of the globules when the temperature inside the shell is lower than the Jeans temperature.

By the conservation of magnetic flux, a magnetic field present in the Universe decreases as a function of the scale factor $\left(B \propto a^{-2}\right)$. On the other hand, during the shell expansion phase, when the first term on the right side of equation (8) is important, the shell expands with a velocity greater than the expansion rate of the Universe. Thus, due to the fact that the field is frozen into the matter, it decreases as $B \propto R_{S}^{-2}$, where $R_{S}$ is the radius of the shock front.

In the last explosion cycle of our model, the seed magnetic field is increased due to the growth of the density contrast of the globules (which may collapse to form quasars and galaxies) in relation to the ambient medium (that is $\delta \rho / \rho \sim 10^{5}$, where $\rho$ is the ambient density). The final collapse can increase the intensity of the seed magnetic field by a factor $\sim 3,000$. Our final (present ) seed magnetic field takes this factor into account.

In table 1 we have the present value of the seed magnetic field produced by the collapse and explosion, at an initial redshift $z_{i}$, of a Population III object with mass $M_{0}$. The evolution in explosion cycles generates large shells of matter with a Compton y-distortion within the limit imposed by the COBE satellite $\left(y \lesssim 1.5 \times 10^{-5}\right.$, see Miranda and Opher 1996, 1997). The explosion models, presented in table 1 , produce voids within the range $18-26 M p c$. The last column of table 1 shows the coherence length $(L)$ of 
the magnetic field. The magnetic field $B$ in the table is for $F=0.01$. For other values of $F$, the magnetic field $B$ is approximately proportional to $F^{-1}$.

\section{DISCUSSION AND CONCLUSIONS}

The results presented in table 1 show that seed magnetic fields with intensity $\sim 10^{-12}-10^{-14} G$ (for values of $F$ within the interval $0.01-1$ ) can naturally be produced in the multicycle explosion scenario, without any previous seed magnetic field in the beginning of the explosions. The intensity of this seed field is sufficiently high to produce, by future dynamo amplification (for example the $\alpha-\omega$ dynamo), a magnetic field with intensity $\sim \mu G$ (on the order of typical galactic magnetic fields). The coherence length $(L)$ of these seed fields is $\sim 1.8 \mathrm{Mpc}$, the typical dimension of clusters of galaxies.

Due to the low values of the seed magnetic fields produced by the previous suggested mechanisms, it is questionable if the dynamo mechanisms have enough time to amplify the suggested seed fields to the observed values. The amplification time scale in the protogalactic disk is of the order of the rotation period of the disk, $\sim 3 \times 10^{8}$ years. Our proposed mechanism creates relatively high values of the magnetic field over a large coherence length so that the dynamo mechanism can be effective.

Because of the large initial magnetic field, the dynamo action needs to operate only over a relatively short time. Comparing with the models previously proposed for the production of magnetic fields, our mechanism produces seeds of the greatest intensity. In this model, the generation of the magnetic field occurs after the recombination era as a result of the formation of the structures of the Universe.

The generation of seed magnetic fields, as a consequence of the explosive scenario for the formation of the large-scale voids, is consistent with the detection of a large-scale intercluster magnetic field, found in the Coma supercluster, with an estimated strength of $0.3-0.6 \mu G$. This detected magnetic field could be the result of a primordial field, generated and amplified during the formation of the supercluster (Kim et al. 1989). It is worth mentioning that coherence of the magnetic field over a large-scale (e.g., the Coma supercluster) could occur via reconnection over causally unconnected regions (Hogan 1983).

It is interesting to note that the magnetic field can have several effects on the history of the Universe (e.g., Kronberg 1994). The question of whether magnetic fields are amplified is relevant in several areas related with the formation and evolution of galaxies (e.g Rees 1978; Coles 1992). For example, stellar formation can change for different values of the magnetic field. That is, the star formation rate and the distribution of stellar masses can be modified, because the magnetic field alters the Jeans mass and is important in the loss of angular momentum during protostellar collapse. Also, as shown by Wasserman (1978), and more recently by Kim et al. (1996), a primordial magnetic field can have a direct effect on triggering galaxy formation. Early creation of magnetic fields can also play an important role in particle cosmology by modifying the clustering properties of dark matter particles sensitive to the presence of a magnetic field (Enqvist 1998).
Table 1. Seed Magnetic Field.

$\begin{array}{lccc}M_{0}\left(M_{\odot}\right) & z_{i} & B(G) & L(M p c) \\ 10^{6} & & & \\ 10^{7} & 290 & 1.2 \times 10^{-12} & 1.6 \\ 10^{8} & 150 & 1.1 \times 10^{-12} & 1.8 \\ 10^{9} & 120 & 1.0 \times 10^{-12} & 2.0 \\ 10^{10} & 70 & 8.5 \times 10^{-13} & 1.5 \\ & 70 & 5.0 \times 10^{-13} & 2.2\end{array}$

The present value of the seed magnetic field $(B)$, and its respective coherence length $(L)$, produced by the collapse and explosion, at initial redshift $z_{i}$, of a Population III object with mass $M_{0}$.

Although in table 1 we present only models that produce large voids with present radii $R_{S F}>18 M p c$, an interesting characteristic of the multiple explosion scenario is that, independent of the radius of the void formed, a seed field can naturally be produced. For example, in the multicycle explosion model, the explosion of an object with initial mass $10^{5} M_{\odot}$ (a typical mass of a globular cluster) at redshift $z=25$ produces a small void with a present diameter $1.5 M p c$ but with a magnetic field of strength $4.5 \times 10^{-10} G$ (with $F=0.01$ ) and a coherence length $\sim 100 k p c$.

A void with radius $1.5 \mathrm{Mpc}$ certainly makes a small contribution to the formation of the large-scale structures of the Universe. But, on the other hand, these small voids formed by explosions can be very important for the generation of seed magnetic fields on galactic scales.

\section{ACKNOWLEDGEMENTS}

We thank Dr. J.C.N. de Araujo for useful discussions. We would also like to thank Dr Alex Lazarian, the referee, for his helpful suggestions and comments that improved the present version of our paper.

O.D.M thanks the Brazilian agency FAPESP for support (grant 97/02596-3). M.O. would also like to thank the Brazilian agency FAPESP for support. R.O. thanks the Brazilian agency $\mathrm{CNPq}$ for partial support. We would also like to thank the Brazilian project Pronex/FINEP (no. 41.96.0908.00) for support. The calculations were made on the Workstation Apollo 9000 purchased by the Brazilian Agency FAPESP.

\section{REFERENCES}

Barrow, J.D., Ferreira, P.G. \& Silk, J. 1997, Phys. Rev. Lett., 78, 3610 .

Biermann, L. 1950, Z. Naturforsch, 5a, 65.

Carr, B.J., Bond, J.R. \& Arnett, W.D. 1984, ApJ, 277, 445.

Carr, B.J. \& Ikeuchi, S. 1985, MNRAS, 213, 497.

Coles, P. 1992, Comm. on Astrop., 16, 45.

Enqvist, K. 1998, astro-ph/9803196.

Harrison, E.R. 1973, MNRAS, 165, 185.

Hogan, C.J. 1983, Phys. Rev. Lett., 51, 1488.

Ikeuchi, S. 1981, PASJ, 33, 211.

Kemp, J.C. 1982, PASP, 94, 627.

Kim, K.T., Kronberg, P.P., Giovannini, G. \& Venturi, T. 1989, Nature, 341, 720.

Kim, E., Olinto, A.V. \& Rosner, R. 1996, ApJ, 468, 28.

Kronberg, P.P. 1994, Rep. Prog. Phys., 57, 325.

Kulsrud, R.M., Cen, R., Ostriker, J.P. \& Ryu, D. 1997, ApJ, 480, 481 . 
Lazarian, A. 1992, A\&A, 264, 326.

Mestel, L. \& Roxburgh, I.W. 1962, ApJ, 136, 615.

Miranda, O.D. \& Opher, R. 1996, MNRAS, 283, 912.

Miranda, O.D. \& Opher, R. 1997, ApJ, 482, 573.

Olesen, P. 1997, hep-ph/9708320

Opher, M. \& Opher, R 1997a, Phys. Rev. D, 56, 3296.

Opher, M. \& Opher, R 1997b, Phys. Rev. Lett., 79, 2628.

Opher, R. \& Wichoski, U. 1997, Phys. Rev. Lett., 78, 787.

Ostriker, J.P. \& Cowie, L.L. 1981, ApJ, 243, L127.

Peebles, P.J.E. 1993, Principles of Physical Cosmology, Princeton U.P., Princeton.

Quashnock, J.M., Loeb, A. \& Spergel, D.N. 1989, ApJ, 344, L49.

Ratra, B. 1992, ApJ, 391, L1.

Rees, M.J. 1978, Phys. Scrip., 17, 371.

Rees, M.J. 1987, QJRAS, 28, 197.

Roxburgh, I.W. 1966, MNRAS, 132, 201.

Sigl, G. \& Olinto, A.V. 1997, Phys. Rev. D, 55, 4582.

Tajima, T., Cable, S., Shibata, K. \& Kulsrud, R.M. 1992, ApJ, 390, 309.

Törnkvist, O. 1997, hep-ph/9707513

Törnkvist, O. 1998, hep-ph/9801286

Turner, M.S. \& Widrow, L.M. 1988, Phys. Rev. D, 37, 2743.

Wasserman, I. 1978, ApJ, 224, 337.

Zel'Dovich, Ya.B., Ruzmaikin, A.A. \& Sokolov, D.D. 1983, Magnetic Fields in Astrophysics, Gordon \& Breach Pub.

This paper has been produced using the Royal Astronomical Society/Blackwell Science $\mathrm{T}_{\mathrm{E}} \mathrm{X}$ macros. 